\title{
Deep near-IR photometry of eight metal-poor globular clusters in the Galactic bulge and halo*
}

\author{
J.-W. Kim ${ }^{1,2}$, A. Kang ${ }^{1,2}$, J. Rhee ${ }^{1,2}$, H.-I. Kim ${ }^{3}$, Y.-C. Kim ${ }^{1}$, D.-G. Kim ${ }^{1,2}$, C. Chung ${ }^{1,2}$, S.-I. Han ${ }^{1,2}$, \\ M.-S. Chun ${ }^{1}$, and Y.-J. Sohn ${ }^{1,2}$
}

\begin{abstract}
1 Department of Astronomy, Institute of Earth Atmosphere Astronomy, Yonsei University, Seoul 120-749, Korea e-mail: sohnyj@yonsei.ac.kr; kjw0704@galaxy.yonsei.ac.kr

2 Center for Space Astrophysics, Yonsei University, Seoul 120-749, Korea

Korea Astronomy and Space Science Institute, Daejeon 305-348, Korea
\end{abstract}

Received 11 February 2006 / Accepted 25 July 2006

\section{ABSTRACT}

\begin{abstract}
High quality $J, H$ and $K^{\prime}$ images are used to investigate the morphological properties of the near-infrared color-magnitude diagrams for five metal-poor bulge globular clusters and three halo clusters. Photometric parameters to describe the RGB shape, i.e., the colors at fixed magnitudes of $M_{K}=M_{H}=(-5.5,-5,-4$ and -3$)$, the magnitudes at fixed colors of $(J-K)_{o}=(J-H)_{o}=0.70$, and the RGB slope, have been measured from the fiducial normal points of the CMDs. We also measured the near-infrared magnitudes of the RGB bump and tip on the luminosity function of the RGB stars for each cluster. The RGB parameters of the observed metal-poor bulge and halo clusters are consistent with the previous empirical relationships between the RGB parameters and the cluster metallicity for metal-rich bulge clusters and halo clusters. The near-infrared magnitudes of the RGB bump and tip are in good agreement with the theoretical prediction of the Yonsei-Yale isochrone.
\end{abstract}

Key words. Galaxy: structure - Galaxy: globular clusters: general - stars: evolution - infrared: stars - techniques: photometric

\section{Introduction}

Globular clusters play a key role in studies of the formation history of the Galaxy since they are equally present from the central bulge to the outer halo. In particular, the Galactic bulge harbors a rich globular cluster population with a broad metallicity distribution that extends from about twice solar to less than one-tenth solar abundance (Ortolani 1999). The bulge as a whole, however, is a high metallicity domain where most stars have near-solar metallicity (Zoccali et al. 2003; McWilliam \& Rich 1994). In this respect, metal-poor globular clusters in the Galactic bulge are rather unique objects embedded in the metal-rich environment. This situation is clearly a result of the Galaxy formation. Thus, by comparing properties of the stellar population of the metal-poor globular clusters in the bulge with those in the halo, one can constrain theories of the formation and early evolution of the Galaxy.

The amount of optical ground-based CCD photometric data for the stellar population in the bulge globular clusters has been growing steadily in recent years (Ortolani et al. 2003, 2000; Barbuy et al. 1999; and references therein). However, such ground measurements are hampered by many difficulties, such as relatively high reddening, internal differential reddening, background crowding by faint main-sequence stars, and foreground contamination from field stars at the Galactic disk toward the bulge. These complications can be reduced by observing at infrared wavelengths, as the extinction in the $K$ band is only

* Based on observations carried out at the Canada-France-Hawaii Telescope, operated by the National Research Council of Canada, the Centre National de la Recherche Scientifique de France, and the University of Hawaii. about 10 percent of that in the $V$ band (Rieke \& Lebofsky 1985). In addition, near-infrared photometry offers several advantages in studying the cool population of the bulge globular clusters, i.e., the red giant branch (RGB), because of its high sensitivity to low temperature. The contrast between the bright red giants and the unresolved blue body of the cluster is enhanced in the near-infrared with respect to visible wavelengths, thus allowing us to survey for bright RGB stars even in the richly populated innermost region of the clusters affected by stellar crowding.

Recently, a homogeneous near-infrared database of color-magnitude diagrams (CMDs) and luminosity functions (LFs) for 28 Galactic globular clusters distributed over a wide metallicity range of $-2.16<[\mathrm{Fe} / \mathrm{H}]<-0.38$ has been presented in the papers of Ferraro et al. (2000), Valenti et al. (2004a,b,c), Sollima et al. (2004) and Valenti et al. (2005). These papers defined a set of near-infrared photometric indices for RGB morphology (i.e., colors, magnitudes and slopes) and the RGB bump and tip to obtain a quantitative description of the RGB as a function of the cluster's metal abundance. Of the 28 clusters, twelve clusters belong to the Galactic bulge and are all relatively metal-rich with $[\mathrm{Fe} / \mathrm{H}]>-1.0$. Davidge (2000) performed broadband and narrowband near-infrared photometric analysis of 20 metal-poor $([\mathrm{Fe} / \mathrm{H}]<-1.3)$ bulge globular clusters to obtain independent distances, reddenings and metallicities. The data used by Davidge (2000) were recorded with a relatively small CTIO $1.5 \mathrm{~m}$ telescope during mediocre seeing conditions of $\sim 1$ '.5 FWHM. As a result, only the top few magnitudes of the RGB could be sampled in each datum, so that accurate CMD morphologies, such as RGB slope, could not be obtained.

We have focused on obtaining a moderately deep homogeneous photometric data set for the metal-poor bulge globular 
clusters in the near-infrared regime. A total of 20 bulge clusters located within $3 \mathrm{kpc}$ of the Galactic center with metallicities $[\mathrm{Fe} / \mathrm{H}]<-1.0$ have been selected from the list of Harris (1996) to secure a complete set of near-infrared photometry. Typical halo clusters also have been considered for direct comparison with the photometric properties of stars in metal-poor bulge globular clusters. In this paper, we report the details of the nearinfrared photometric study of stars in a sample of five metal-poor bulge clusters (NGC 6541, NGC 6642, NGC 6681, NGC 6717, NGC 6723) and three halo clusters (M3, NGC 5897, M 13), all of which were observed during the same run of the CanadaFrance-Hawaii Telescope (CFHT) in June, 2004. We note here that the origin of the metal-poor bulge globular clusters is still a subject of debate since accurate measurements of kinematics are lacking for all of them. Thus the position of the observed metalpoor bulge clusters might not mean that they originally formed in the bulge. Instead, most of metal-rich globular clusters have been confirmed to be of the bulge population from their kinematics based on measurements of proper motion and radial velocity (e.g., Dinescu et al. 2003; Côte 1999; Minniti 1996).

The results of the RGB morphology for the metal-poor clusters are compared with the previous calibrations for the RGB morphology of the halo globular clusters and bulge clusters with high metallicities (e.g., Valenti et al. 2005, 2004a,b). The observations and the procedures used to reduce the data are described in Sect. 2. In Sect. 3, we describe the properties of the observed near-infrared CMDs and the derived fiducial normal points of the CMDs. In Sect. 4, we present the morphological properties of the observed near-infrared CMDs including the RGB shape feature, RGB bump and tip. A summary of the results follows in Sect. 5.

\section{Observations, data reduction, and photometric measurements}

The images of target clusters were obtained during the nights of UT June 3-4, 2004 using the CFHTIR infrared imager mounted on the $\mathrm{f} / 8$ Cassegrain focus of the $3.6 \mathrm{~m}$ CFHT. The CFHTIR contains a $1024 \times 1024 \mathrm{Hg}$ :Cd:Te array with an angular scale of $0.211 /$ pixel. Thus each image covers a total field-ofview of 3. $6 \times 3.6$ on the sky. Data were recorded through $J, H$ and $K^{\prime}$ filters for the central region of the clusters, and the observations for each filter were split between short and long exposures in order to optimize the photometry of stars at the bright upper RGB and faint lower main-sequence turn-off (MSTO) regions. The total exposure times of the short and long exposures were $8 \mathrm{~s}$ and $240 \mathrm{~s}$ each per filter for the complete observing sequence of four 2-s and eight 30-s exposures, respectively. Only short exposures were secured for the observations of M3. A four-point square dither pattern was used to assist with the identification and rejection of bad pixels and cosmic rays as well as the construction of on-sky calibration frames. The UKIRT standard stars were observed during both nights for photometric calibration. The summary of the run is presented in Table 1 .

Calibration frames (darks, flats, and blank sky backgrounds) were obtained from the observations. Dark frames for each integration time were recorded at the beginning and the end of the run. Dome flats were constructed by subtracting exposures of the dome white spot taken with the lamps off from those recorded with the lamps on. Thermal emission patterns for each filter were constructed by median combined flat-fielded images of blank sky regions, which were recorded at various times throughout the run.
Table 1. Observational log.

\begin{tabular}{ccccc}
\hline \hline Region & Target & Filter & Exp. time $(\mathrm{s})$ & $F W H M\left({ }^{\prime \prime}\right)$ \\
\hline Halo & NGC 5272 & $J$ & $4 \times 2$ & 0.56 \\
& (M 3) & $H$ & $4 \times 2$ & 0.51 \\
& & $K^{\prime}$ & $4 \times 2$ & 0.54 \\
& NGC 5897 & $J$ & $4 \times 2,8 \times 30$ & $0.66,0.70$ \\
& & $H$ & $4 \times 2,8 \times 30$ & $0.73,0.72$ \\
& NGC 6205 & $K^{\prime}$ & $4 \times 2,8 \times 30$ & $0.71,0.77$ \\
& (M 13) & $H$ & $4 \times 2,8 \times 30$ & $0.74,0.72$ \\
& & $K^{\prime}$ & $4 \times 2,8 \times 30$ & $0.69,0.70$ \\
Bulge & NGC 6541 & $J$ & $4 \times 2,8 \times 30$ & $0.69,0.64$ \\
& & $H$ & $4 \times 2,8 \times 30$ & $1.14,1.00$ \\
& & $K^{\prime}$ & $4 \times 2,8 \times 30$ & $0.94,0.89$ \\
& NGC 6642 & $J$ & $4 \times 2,8 \times 30$ & $0.71,0.73$ \\
& & $H$ & $4 \times 2,8 \times 30$ & $0.74,0.76$ \\
& NGC 6681 & $K^{\prime}$ & $4 \times 2,8 \times 30$ & $0.75,0.76$ \\
& & $H$ & $4 \times 2,8 \times 30$ & $0.69,0.69$ \\
& & $K^{\prime}$ & $4 \times 2,8 \times 30$ & $0.63,0.65$ \\
& NGC 6717 & $J$ & $4 \times 2,8 \times 30$ & $0.66,0.64$ \\
& & $H$ & $4 \times 2,8 \times 30$ & $0.65,0.73$ \\
& & $K^{\prime}$ & $4 \times 2,8 \times 30$ & $0.65,0.69$ \\
& NGC 6723 & $J$ & $4 \times 2,8 \times 30$ & $0.73,0.70$ \\
& & $H$ & $4 \times 2,8 \times 30$ & $0.71,0.71$ \\
& & $K^{\prime}$ & $4 \times 2,8 \times 30$ & $0.68,0.70$ \\
\hline
\end{tabular}

The data were reduced using a standard sequence for nearinfrared images: (1) subtracting a dark frame; (2) dividing by dome flats; (3) subtracting the sky background level, which was estimated by taking the mode of the pixel intensity distribution in each flat-fielded frame; and (4) subtracting the thermal signature. The processed images were aligned to correct the dither offsets, then median-combined to have $8 \mathrm{~s}$ and $240 \mathrm{~s}$ exposure times for each short and long exposure. Typical seeings, as measured from the reduced images, are less than 0.8 FWHM, except for NGC $6541\left(\sim 1^{\prime \prime} 0\right)$. The relatively poor seeing value of NGC 6541 was due to the large airmass $\sim 2.5$ during the run because of its low declination (i.e., $\delta \sim-43$ ).

The brightnesses of individual objects in the target clusters were measured with the point-spread function fitting routine DAOPHOT II/ALLSTAR (Stetson 1987; Stetson \& Harris 1988). Faint stars were detected only in images with long exposures. On the other hand, the brightnesses of stars around the RGB tip were measured only in images with short exposures because of saturation in the long-exposure images. For stars detected in both the short and long exposures, the measurement with the smaller detection error was assigned as the brightness. To limit the number of false detections, only those objects detected in all three filters and with detection errors less than 0.2 mag were considered for the photometric analysis. The calibration equations obtained from the standard stars were then applied to the magnitudes of the stars in the target clusters, neglecting the color term due to the small color range of the nearinfrared standard stars. We note that the near-infrared photometry of stars in the selected nearby dwarf galaxies (Sohn et al. 2006; Kang et al. 2005), for which the images were obtained on the same nights with same instrument, showed that the photometric calibration is consistent with results from other nearinfrared photometry. Here, we also note that the central wavelength of $2.12 \mu \mathrm{m}$ for the $K^{\prime}$ filter of CFHTIR is slightly shorter than those of the standard $K(2.20 \mu \mathrm{m})$ and $K_{\mathrm{S}}(2.15 \mu \mathrm{m})$ filters. This leads to a lower thermal component of the sky background in $K^{\prime}$ and deeper imaging with the same integration time than 


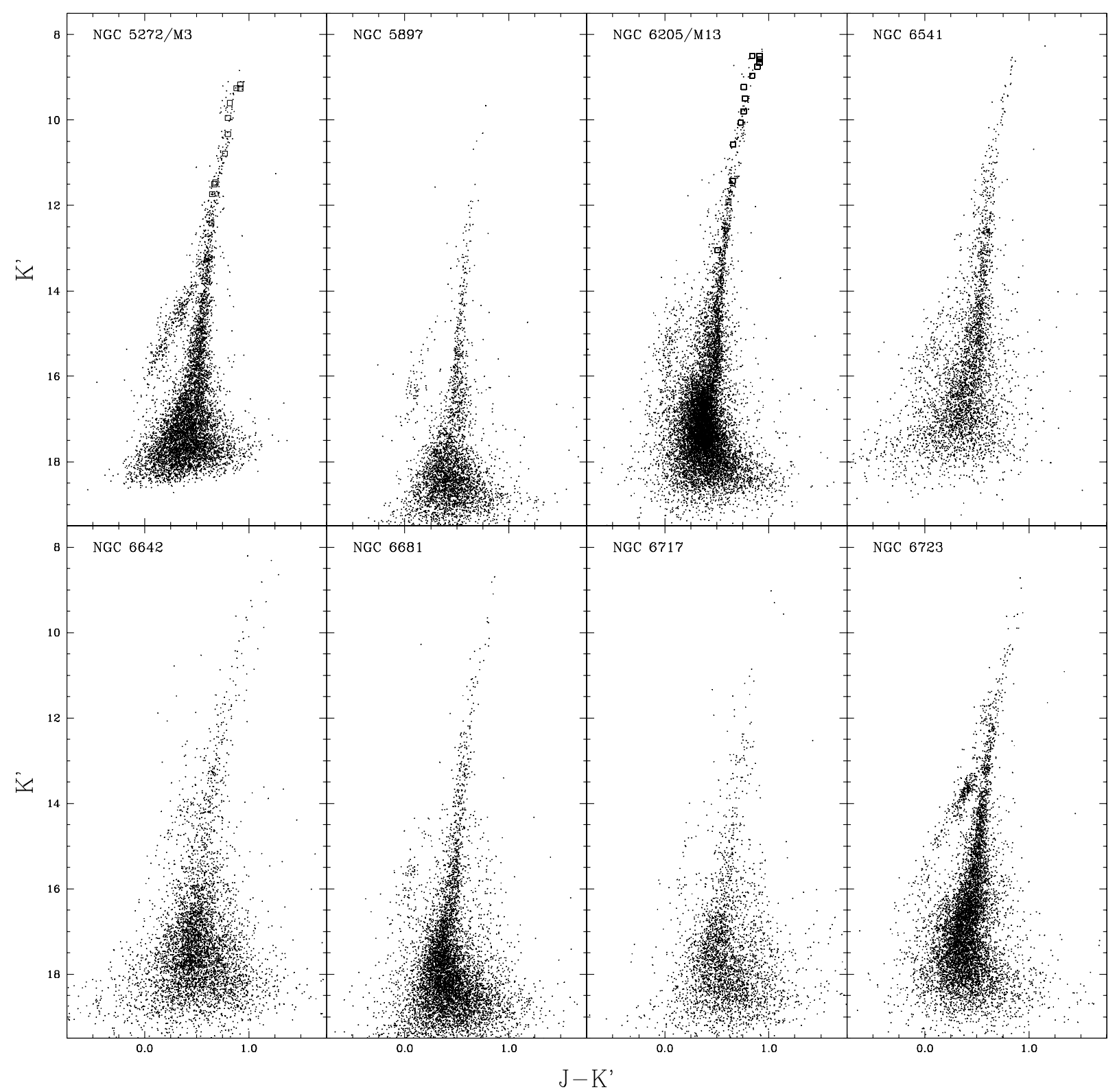

Fig. 1. $(J-K, K)$ CMDs of three halo clusters (M3, NGC 5897, M 13) and five bulge clusters (NGC 6541, NGC 6642, NGC 6681, NGC 6717, NGC 6723). Open squares indicate the measurements of Cohen et al. (1978) for bright giants in the outer regions of M 3 and M 13.

in the standard $K$ and $K_{\mathrm{S}}$ filters (Simons \& Tokunaga 2002; Tokunaga et al. 2002; Wainscoat \& Cowie 1992).

\section{Color-magnitude diagrams}

\subsection{Photometric properties}

Figures 1 and 2 show near-infrared $\left(J-K^{\prime}, K^{\prime}\right)$ and $(J-H$, $H$ ) CMDs of the resolved stars in the central 3.6 $6 \times 3.6$ area for the observed eight metal-poor globular clusters in the Galactic halo and bulge. While the CMD of M3 shows a slightly saturated pattern for the brightest RGB stars in the $H$ band, all of the observations are deep enough to detect the base of the RGB at $\Delta K^{\prime} \sim \Delta H \approx 8 \mathrm{mag}$ fainter than the RGB tip, and $\sim 4$ mag below the HB. The sub-giant branch (SGB) sequence and MSTO also are detectable in the CMDs of most of the observed clusters. The slanted HB sequences are well separated from the RGB for all clusters, as we expected for the typical morphology of metal-poor globular clusters. Scattering in the CMDs of bulge clusters may be due to contamination by foreground field stars toward the Galactic bulge.

Comparisons with published near-infrared photometric measurements verify the calibration of the CFHTIR data. The open squares in Figs. 1 and 2 are the measurements by Cohen et al. (1978) for bright giants in the outer regions of M 3 and M 13. While our near-infrared CMDs contain stars in the central region of the clusters, in which crowding and internal differential reddening of stars are expected, both photometries seem to overlap in the CMDs. Also, the comparison of our CMDs of M3 and M 13 with the mean ridge lines in Davidge \& Courteau (1999) derived from CFHT AOB observations indicates a good agreement with each other. For an arbitrary sample of 10 bright RGB stars in M 13, a direct star-to-star comparison between the photometric data in this paper and Valenti et al. (2004c) shows small offsets of $\Delta K=0.03 \pm 0.01$ and $\Delta(J-K)=0.04 \pm 0.01$. The offsets, which are due to different the photometric systems 


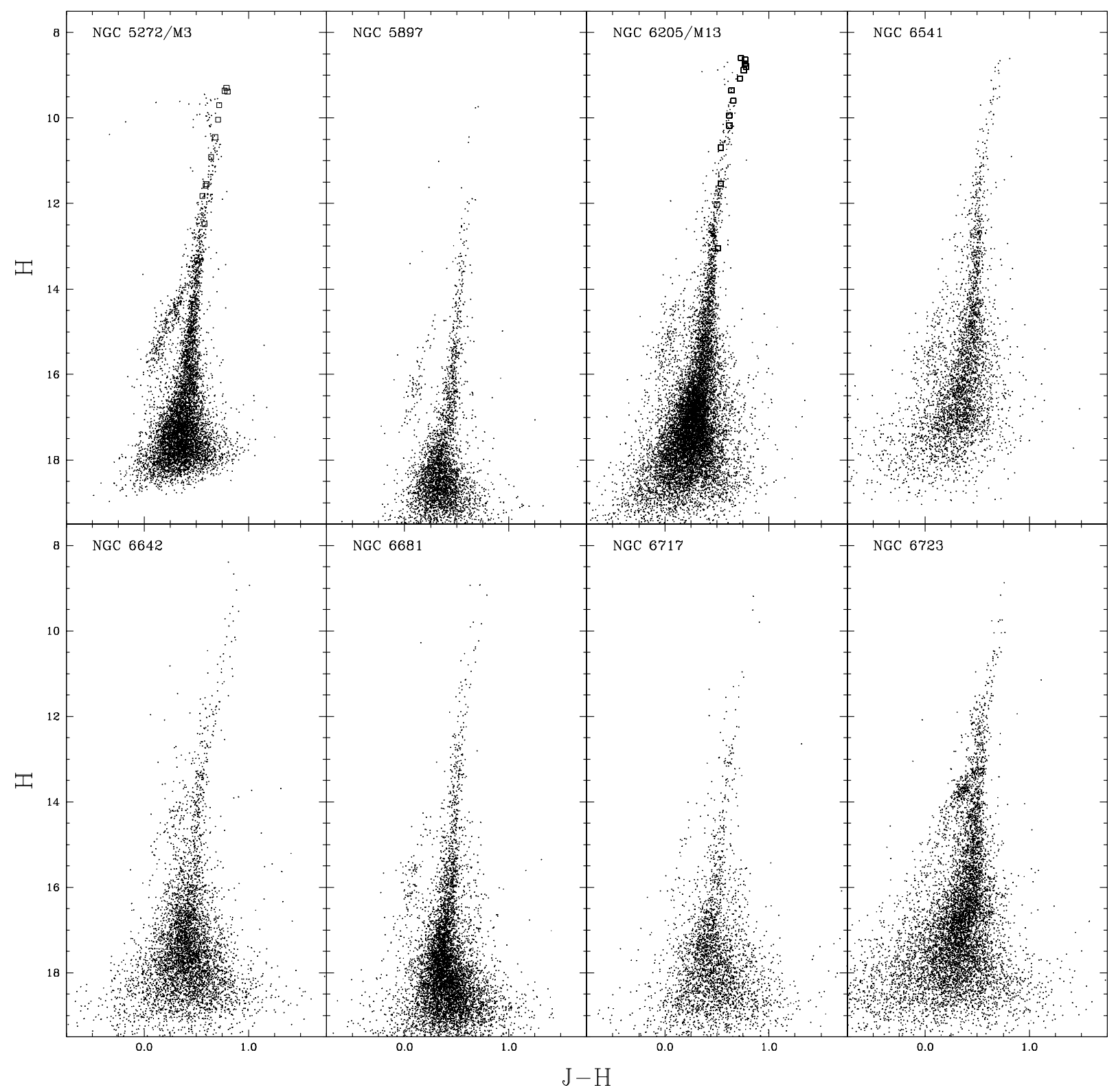

Fig. 2. $(J-H, H)$ CMDs of three halo clusters (M3, NGC 5897, M 13) and five bulge clusters (NGC 6541, NGC 6642, NGC 6681, NGC 6717, NGC 6723). Open squares indicate the measurements of Cohen et al. (1978) for bright giants in the outer regions of M 3 and M 13.

of the UKIRT and 2MASS, become negligible after transformation into the 2MASS photometric system (cf. Sect. 3.3). Thus, it is apparent that the current near-infrared photometry from the CFHTIR observation is in reasonable agreement with other published near-infrared photometry for globular clusters.

\subsection{Metallicity, distance modulus, and reddening}

For the calibration of the RGB morphology parameters to the metal abundances of globular clusters, Valenti et al. (2005, 2004a) used the metallicity $[\mathrm{Fe} / \mathrm{H}]$ in the Carreta \& Gratton (1997, CG97) scale when available, otherwise they transformed the metallicity of the Zinn (1985) scale by using Eq. (7) of CG97. We adopt a metallicity $[\mathrm{Fe} / \mathrm{H}]_{\mathrm{CG} 97}$ and global metallicity $[\mathrm{M} / \mathrm{H}]$ of the observed globular clusters from Ferraro et al. (1999) to directly compare the photometric properties of the measured RGB morphology with the results of Valenti et al. (2004a). However, Ferraro et al. (1999) lack data for three bulge clusters in this study (NGC 6541, NGC 6642, and NGC 6723). For the three clusters, we obtained $[\mathrm{Fe} / \mathrm{H}]_{\mathrm{CG} 97}$ by transforming the data given in Zinn (1985) into the scale of CG97 as per Valenti et al. (2004a). For the same three clusters, we estimated $[\mathrm{M} / \mathrm{H}]$ by using the equation for the $\alpha$-elements enhanced theoretical evolutionary sequence (Salaris et al. 1993), i.e., $[\mathrm{M} / \mathrm{H}]=$ $[\mathrm{Fe} / \mathrm{H}]_{\mathrm{CG} 97}+\log \left(0.638 f_{\alpha}+0.362\right)$ with $f_{\alpha}=10^{0.28}$, where $f_{\alpha}$ is the enhancement factor of the $\alpha$-elements. The adopted and estimated metallicity and global metallicity are listed in Cols. 2 and 3 of Table 2.

In a similar fashion, we adopt the distance moduli of five clusters from Ferraro et al. (1999), in which a new methodology is presented to derive the actual level of the zero-age horizontal branch (ZAHB) and the distance moduli from the matching of $V_{\text {ZAHB }}$ and the theoretical models (Straniero et al. 1997). For the other three bulge clusters (NGC 6541, NGC 6642, and NGC 6723), a similar procedure to Ferraro et al. (1999) has been applied to determine the distance moduli of each cluster from 
Table 2. Adopted and estimated metallicity, distance modulus, reddening, and extinction values of the three halo clusters and five bulge clusters observed.

\begin{tabular}{lcccccccc}
\hline \hline Target & {$[\mathrm{Fe} / \mathrm{H}]_{\mathrm{CG} 97}$} & {$[\mathrm{M} / \mathrm{H}]$} & $\mu_{o}$ & $E(B-V)$ & $A_{J}$ & $A_{H}$ & $A_{K}$ & note \\
\hline M3 & -1.34 & -1.16 & 15.03 & 0.01 & 0.009 & 0.006 & 0.004 & halo \\
NGC 5897 & -1.59 & -1.44 & 15.64 & 0.09 & 0.081 & 0.052 & 0.033 & $\prime \prime$ \\
M 13 & -1.39 & -1.18 & 14.43 & 0.02 & 0.018 & 0.012 & 0.007 & $\prime \prime$ \\
NGC 6541* & -1.62 & -1.42 & 14.04 & 0.14 & 0.126 & 0.081 & 0.051 & bulge \\
NGC 6642 & -1.08 & -0.88 & 14.49 & 0.41 & 0.370 & 0.236 & 0.150 & $\prime \prime$ \\
NGC 6681 & -1.27 & -1.07 & 15.00 & 0.07 & 0.063 & 0.040 & 0.026 & $\prime \prime$ \\
NGC 6717 & -1.10 & -0.90 & 14.43 & 0.22 & 0.198 & 0.127 & 0.081 & $\prime \prime$ \\
NGC 6723* & -0.93 & -0.73 & 14.72 & 0.05 & 0.045 & 0.029 & 0.018 & $"$ \\
\hline
\end{tabular}

${ }^{*}$ Metallicity is estimated by using equations in Carreta \& Gratton (1997) with [Fe/H] in Zinn (1985), and distance modulus is estimated from the matching of $V_{\mathrm{ZAHB}}$ and the theoretical HB synthetic model as per Ferraro et al. (1999). Metallicity and distance moduli of the other clusters are adopted from Ferraro et al. (1999).

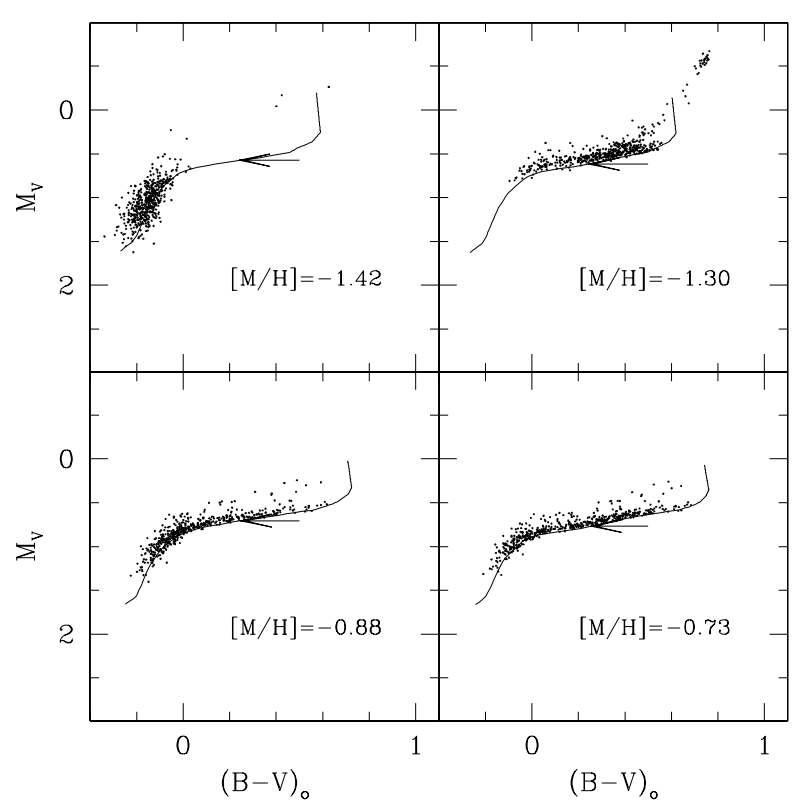

Fig. 3. Generated synthetic HBs with $[\mathrm{M} / \mathrm{H}]=-1.42,-1.30,-0.88$, and -0.73 . The solid lines indicate ZAHB and dots are the generated HBs in each panel. The arrow indicates the theoretical ZAHB level at $\log T_{\text {eff }}=3.85$.

the ZAHB levels. In this paper, the synthetic HBs for each cluster have been generated using the method of Lee et al. (1994) with the HB evolutionary tracks of Yi et al. (1997). To directly compare with the corresponding results in Fig. 3 of Ferraro et al. (1999), we generated a synthetic HB with $[\mathrm{M} / \mathrm{H}]=-1.3$. The color and magnitude levels of the ZAHB at $\log T_{\text {eff }}=3.85$ are $(B-V)=0.243$ and $M_{V}=0.617$, which are in good agreement with Ferraro et al. (1999). In Fig. 3, we also present synthetic HBs of cases with $[\mathrm{M} / \mathrm{H}]=-1.42,-0.88$, and -0.73 for the clusters NGC 6541, NGC 6642, and NGC 6723, respectively. The levels of the ZAHB at $\log T_{\text {eff }}=3.85$ are $M_{V}=0.575,0.705$, and 0.769 for each case. The synthetic HBs are now shifted in magnitude and color to match the observed HBs in the CMDs of NGC 6541 (Alcaino 1997), NGC 6642 (Barbuy et al. 2006), and NGC 6723 (Alcaino 1999). On the line indicating the theoretical ZAHB level at $\log T_{\text {eff }}=3.85$, the observed $V_{\text {ZAHB }}$ values are then read to be $15.07 \pm 0.12,16.56 \pm 0.17$, and $15.66 \pm 0.12$, respectively, for the three clusters. The errors in $V_{\mathrm{ZAHB}}$ have been estimated by combining the uncertainty of multiple eye fits of ZAHB level and the photometric error at the HB level in each cluster.
The reddening and extinction correction in the near-infrared has been computed by adopting the latest compilation of $E(B-V)$ by Harris (1996) and by applying the reddening ratios of Schlegel et al. (1998). The reddening and extinction values in the near-infrared for each cluster are listed in Cols. 5-8 of Table 2. Now, the distance moduli are estimated from the ZAHB level in synthetic HB and the observed ZAHB level, taking into account the extinction value of each cluster. The determined distance moduli of NGC 6541, NGC 6642, and NGC 6723 are listed in Col. 4 of Table 2.

\subsection{The fiducial normal points}

Ferraro et al. (2000) suggested a simple standard procedure to compute the fiducial ridge line for the RGB sequence in CMDs: first, make an eye-fit selection of candidate RGB stars, and second, compute a low-order polynomial fit to the selected RGB stars, rejecting those $> \pm 2 \sigma$ from the best fit line. However, due to the curvatures of the RGB, the SGB and the MS, a simple polynomial function will not give a good fit over the whole magnitude range of the moderately deep CMDs for the clusters observed here. For the current study, we define the normal points of the RGB, the SGB and the MS as the mean magnitude and color in the $0.25 \mathrm{mag}$ bin of the CMDs in Figs. 1 and 2. Making this calculation requires two steps. First, we determine the mean magnitude and color in the bin, excluding asymptotic giant branch(AGB) stars, vertical HB stars and foreground field stars by eye. Second, rejecting stars with colors larger than $\pm 2 \sigma$ of the mean, the mean values of the magnitude and color are calculated again for stars within the $\pm 0.25 \mathrm{mag}$ interval. The procedure with a $2 \sigma$ rejection criterion is iterated until the mean values of magnitude and color are stable at constant values, to which we assign the fiducial normal points of the CMDs.

The estimated normal points are converted into the absolute plane in color and magnitude using the adopted reddening and distance scale for each observed cluster. In order to properly compare the near-infrared photometric properties of globular clusters in this paper with those in the previous homogeneous calibrations (e.g., Valenti et al. 2005, 2004a,b), we transformed the color and magnitude of the normal points in the UKIRT system into the 2MASS system by using the Eqs. (37)-(39) of Carpenter (2001). Figure 4 shows the transformed fiducial normal points of the observed clusters in $(J-K)_{o}-M_{K}$ and $(J-H)_{o}-M_{H}$ planes, from which we will derive the photometric indices of the RGB. Note that stars near the RGB tip of M 3 are saturated in the $(J-H)_{o}-M_{H}$ plane, for which we estimated the fiducial normal points from the 2MASS catalog data of the 

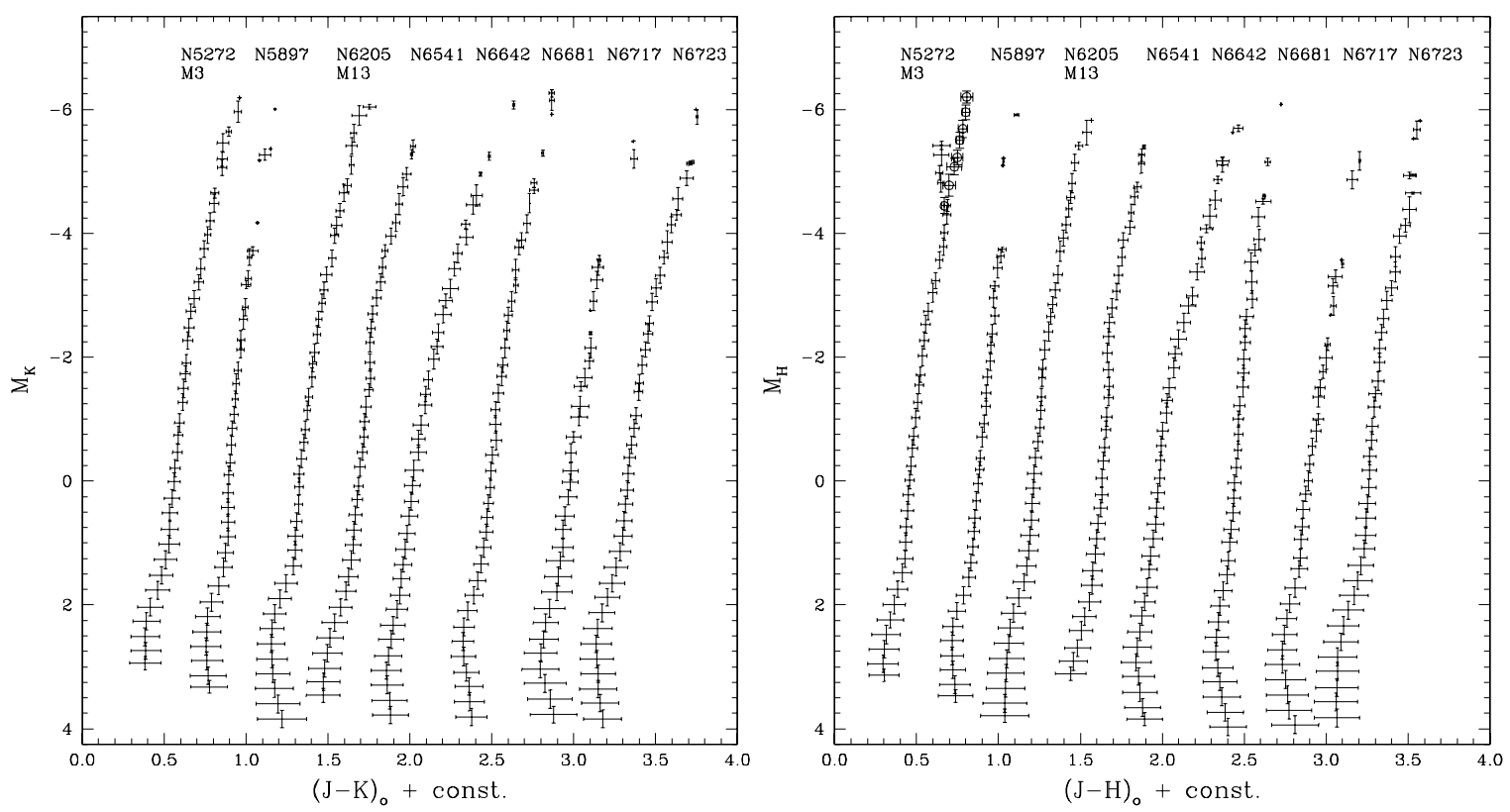

Fig. 4. Fiducial normal points of target clusters in $(J-K)_{o}-M_{K}$ and $(J-H)_{o}-M_{H}$ planes. For clarity, the colors are given zero-point offsets; from left to right, these are const. $=0.0,0.4,0.8,1.2,1.6,2.0,2.4$ and 2.8 mag. Open circles indicate fiducial normal points of M 3 determined from the 2MASS data.

same area observed in the current study, and shown in Fig. 4 by open circles.

\section{Morphology of the near-infrared CMDs}

\subsection{The RGB location and slope}

While several parameters describing the RGB location in color and magnitude have been suggested (e.g., Ferraro et al. 1999; Da Costa \& Armandroff 1990; Frogel et al. 1983), Ferraro et al. (2000) defined a new set of photometric indices in the nearinfrared and optical CMDs to properly characterize the overall behavior of the RGB as a function of cluster metallicity. Recently, Valenti et al. (2004a) presented an updated calibration of the various RGB indices of Ferraro et al. (2000) in terms of cluster metallicity. For a direct comparison with the RGB morphology of 28 clusters from Valenti et al. (2005, 2004a), in the present study we measure the photometric color and magnitude indices for the RGB morphology of the eight observed clusters in near-infrared CMDs. These are the intrinsic colors in $(J-K)_{o}$ and $(J-H)_{o}$ corresponding to four different magnitude levels of $M_{K}=M_{H}=(-5.5,-5,-4$ and -3$)$ and the magnitudes of $M_{K}$ and $M_{H}$ at fixed colors of $(J-K)_{o}=(J-H)_{o}=0.7$.

To derive these parameters for the RGB location of each cluster, namely colors at fixed magnitudes and magnitudes at fixed colors, we applied a second- or third-order polynomial fit to adjacent $\gtrsim 10$ fiducial normal points of CMDs (Fig. 4) at the given magnitude and color. Table 3 lists the measured photometric indices with the measurement errors, describing the RGB location in color and magnitude for the eight observed clusters.

Figures 5 and 6 show mean colors at fixed magnitudes of $M_{K}=M_{H}=(-5.5,-5,-4$ and -3$)$ as functions of metallicity $[\mathrm{Fe} / \mathrm{H}]_{\mathrm{CG} 97}$ and global metallicity $[\mathrm{M} / \mathrm{H}]$ for the observed three halo and five metal-poor bulge clusters. Filled triangles and circles represent the measurements for the three halo clusters and five bulge clusters observed here. Vertical bars represent the previous measurements of the halo clusters and the metalrich bulge clusters of Valenti et al. (2005, 2004a). Solid lines are the calibration relations of Valenti et al. (2004a). In Figs. 5 and 6, the trends of RGB color indices of $(J-K)_{o}$ and $(J-H)_{o}$ as a function of metallicity agree well with the calibration relations of Valenti et al. (2004a) for the observed halo clusters and metal-rich bulge clusters. The fitted slopes increase progressively toward the RGB tip, and the RGB color indices are bluer for the metal-poor clusters than metal-rich clusters, as expected in Valenti et al. $(2005,2004 a)$.

Figure 7 shows the dependence of the absolute magnitudes of $M_{K}$ and $M_{H}$ at fixed colors of $(J-K)_{o}=(J-H)_{o}=0.70$ on the metallicity of the observed clusters. It is apparent that the results given in Fig. 7 for the eight metal-poor bulge and halo clusters seem to be consistent with previous calibrations for halo clusters and metal-rich bulge globular clusters (Valenti et al. 2004a). However, the distribution of the $M_{H}$ magnitudes with cluster metallicity for the observed clusters show a larger deviation to the linear scaling calibration relations than the halo clusters and metal-rich bulge clusters of Valenti et al. (2005, 2004a). This may be due to the uncertainty associated with the determination of the magnitude of the RGB at a fixed color, which is caused mainly by uncertainties in the reddening and the distance modulus in addition to the polynomial fitting measurement errors on the fiducial normal points. Indeed, Valenti et al. (2004a) noted that an error of a few hundredths of magnitude in the reddening correction easily implies $\sim 0.2$ mag uncertainty in the absolute magnitudes.

For the near-infrared photometry, the RGB slope usually has been determined by fitting an equation of the form $J-K=b+a K$ to the upper part of the RGB in the $(J-K, K) \mathrm{CMD}$, where the RGB morphology is less curved than in any other plane (e.g., Valenti et al. 2004a; Ferraro et al. 2000; Kuchinski et al. 1995; Kuchinski \& Frogel 1995). The RGB slope in a CMD is independent of reddening and distance. Moreover, the RGB slope becomes progressively flatter with increasing metallicity, probably because enhanced molecular blanketing could result in redder colors at constant temperature in the coolest and brightest stars (Kuchinski et al. 1995; Ortolani et al. 1991). Therefore, the 
Table 3. Photometric parameters of the RGB morphology for the observed clusters.

\begin{tabular}{ccccccccc}
\hline \hline Name & M3 & NGC 5897 & M 13 & NGC 6541 & NGC 6642 & NGC 6681 & NGC 6717 & NGC 6723 \\
\hline$(J-K)_{o}^{-5.5}$ & $0.879 \pm 0.02$ & $0.757 \pm 0.02$ & $0.856 \pm 0.03$ & $0.824 \pm 0.02$ & $0.924 \pm 0.03$ & $0.826 \pm 0.02$ & $0.968 \pm 0.03$ & $0.940 \pm 0.02$ \\
$(J-K)_{o}^{-5.0}$ & $0.840 \pm 0.02$ & $0.727 \pm 0.02$ & $0.828 \pm 0.03$ & $0.789 \pm 0.02$ & $0.843 \pm 0.03$ & $0.778 \pm 0.02$ & $0.887 \pm 0.03$ & $0.899 \pm 0.02$ \\
$(J-K)_{o}^{-4.0}$ & $0.766 \pm 0.03$ & $0.657 \pm 0.02$ & $0.747 \pm 0.02$ & $0.687 \pm 0.02$ & $0.720 \pm 0.03$ & $0.686 \pm 0.02$ & $0.777 \pm 0.03$ & $0.787 \pm 0.03$ \\
$(J-K)_{o}^{-3.0}$ & $0.688 \pm 0.03$ & $0.601 \pm 0.02$ & $0.673 \pm 0.02$ & $0.595 \pm 0.02$ & $0.632 \pm 0.04$ & $0.628 \pm 0.02$ & $0.714 \pm 0.03$ & $0.696 \pm 0.03$ \\
& & & & & & & & \\
$(J-H)_{o}^{-5.5}$ & $0.765 \pm 0.03$ & $0.690 \pm 0.02$ & $0.724 \pm 0.02$ & $0.695 \pm 0.02$ & $0.816 \pm 0.03$ & $0.680 \pm 0.03$ & $0.820 \pm 0.02$ & $0.751 \pm 0.03$ \\
$(J-H)_{o}^{-5.0}$ & $0.729 \pm 0.03$ & $0.667 \pm 0.02$ & $0.667 \pm 0.02$ & $0.663 \pm 0.02$ & $0.759 \pm 0.03$ & $0.644 \pm 0.03$ & $0.796 \pm 0.02$ & $0.717 \pm 0.03$ \\
$(J-H)_{o}^{-4.0}$ & $0.671 \pm 0.03$ & $0.623 \pm 0.02$ & $0.602 \pm 0.02$ & $0.584 \pm 0.02$ & $0.659 \pm 0.03$ & $0.584 \pm 0.03$ & $0.727 \pm 0.02$ & $0.665 \pm 0.04$ \\
$(J-H)_{o}^{-3.0}$ & $0.594 \pm 0.03$ & $0.583 \pm 0.02$ & $0.539 \pm 0.03$ & $0.511 \pm 0.03$ & $0.578 \pm 0.04$ & $0.537 \pm 0.03$ & $0.650 \pm 0.02$ & $0.580 \pm 0.03$ \\
$M_{K}^{(J-K)_{o}=0.7}$ & $-3.182 \pm 0.41$ & $-4.530 \pm 0.22$ & $-3.435 \pm 0.33$ & $-4.140 \pm 0.23$ & $-3.710 \pm 0.28$ & $-4.102 \pm 0.25$ & $-2.730 \pm 0.33$ & $-3.045 \pm 0.37$ \\
$M_{H}^{(J-H)_{o}=0.7}$ & $-4.553 \pm 0.45$ & $-5.734 \pm 0.44$ & $-5.317 \pm 0.20$ & $-5.558 \pm 0.34$ & $-4.508 \pm 0.37$ & $-5.936 \pm 0.24$ & $-3.656 \pm 0.25$ & $-4.873 \pm 0.50$ \\
$R G B_{\text {slope }}$ & $-0.073 \pm 0.003$ & $-0.047 \pm 0.004$ & $-0.069 \pm 0.003$ & $-0.072 \pm 0.003$ & $-0.104 \pm 0.006$ & $-0.075 \pm 0.003$ & $-0.077 \pm 0.004$ & $-0.082 \pm 0.003$ \\
\hline
\end{tabular}

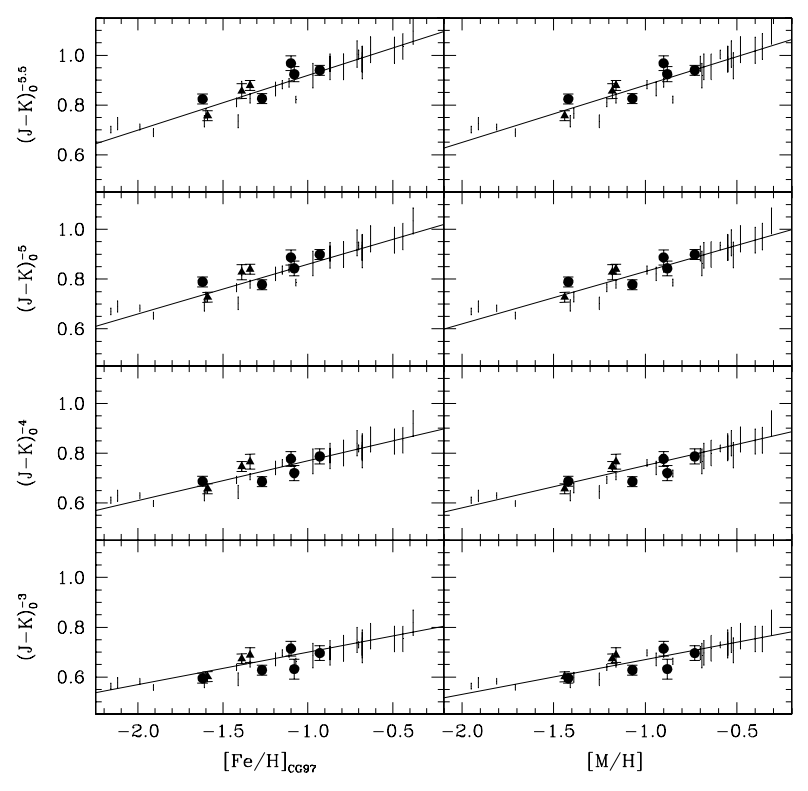

Fig. 5. RGB $(J-K)_{o}$ color indices at fixed magnitudes of $M_{K}$ as a function of the metallicity $[\mathrm{Fe} / \mathrm{H}]_{\mathrm{CG} 97}$ and the global metallicity $[\mathrm{M} / \mathrm{H}]$. Filled triangles and circles represent three halo clusters and five metalpoor bulge clusters observed here, respectively. Vertical bars represent the halo clusters and the metal-rich bulge clusters of Valenti et al. (2005, 2004a). Solid lines are the calibration relations of Valenti et al. (2004a).

RGB slope of the CMD morphology is useful indicator of cluster metallicity.

To obtain the best possible measurements for the RGB slope, contamination of field stars and AGB stars must be analyzed and eliminated. Valenti et al. (2004a) used the 2MASS catalog to select the control field of clusters, and then randomly removed the estimated number of field stars from the cluster RGB sample. Ferraro et al. (2000) used the RGB samples within the criteria of the polynomial fit to determine the fiducial ridge line of the RGB. In this paper, we used the fiducial normal points (Sect. 3.3) which were determined by using the RGB samples within $2 \sigma$ deviation of the mean color at the given magnitude bin. This process statistically removed the AGB, HB and field stars from the CMD.

To determine the RGB slope, Kuchinski et al. (1995) included stars on the RGB that are between 0.6 and $5.1 \mathrm{mag}$

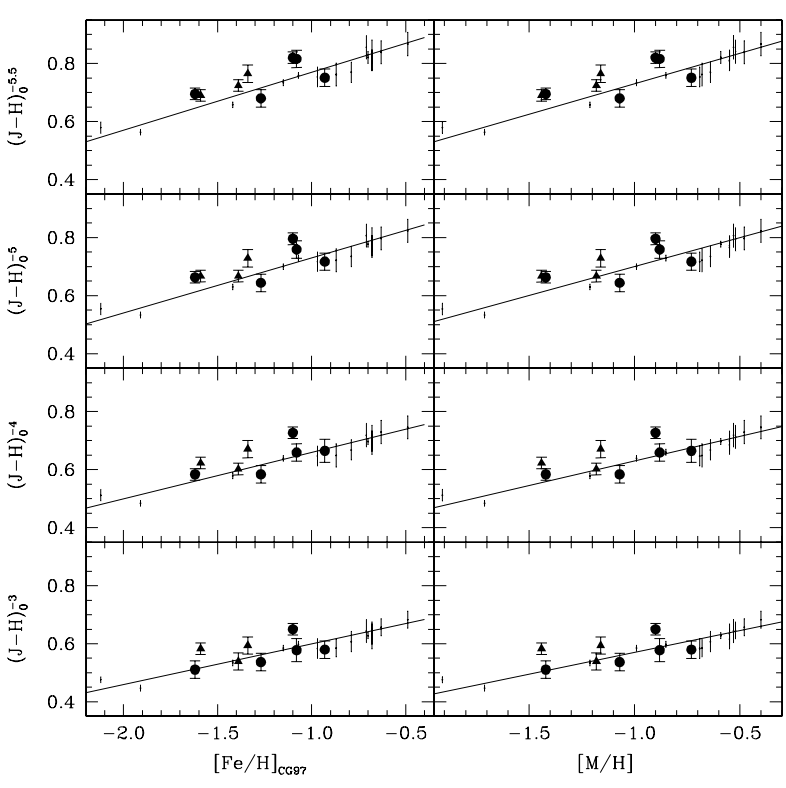

Fig. 6. RGB $(J-H)_{o}$ color indices at fixed magnitudes of $M_{H}$ as a function of the metallicity $[\mathrm{Fe} / \mathrm{H}]_{\mathrm{CG} 97}$ and the global metallicity $[\mathrm{M} / \mathrm{H}]$. Symbols are the same as Fig. 5.

brighter than the ZAHB in $K$. Valenti et al. (2004a) fit the RGB in a magnitude range between 0.5 and 5 mag fainter than the brightest star of each cluster to avoid the almost impossible measurement of the location of the ZAHB in the near-infrared CMD for the case of the low-intermediate-metallicity cluster. In this paper, we used the magnitude range between 0 and 5 mag fainter than the brightest point of the fiducial normal points to determine the RGB slope for each cluster.

The derived values of RGB slopes for the three halo clusters and five metal-poor bulge clusters are listed in the bottom of Table 3. Figure 8 shows the RGB slopes as a function of metallicity with the data from Valenti et al. (2004a). We also plot in Fig. 8 the calibrated relations of the RGB slope versus the metallicity $[\mathrm{Fe} / \mathrm{H}]_{\mathrm{CG} 97}$ and the global metallicity $[\mathrm{M} / \mathrm{H}]$ found by Valenti et al. (2004a) and Ivanov \& Borissova (2002). It is apparent in Fig. 8 that, for the observed halo and metal-poor bulge clusters, the dependency of the RGB slopes on the metallicity at the given magnitude range is consistent with the previous results, i.e., the RGB slopes become progressively steeper with 


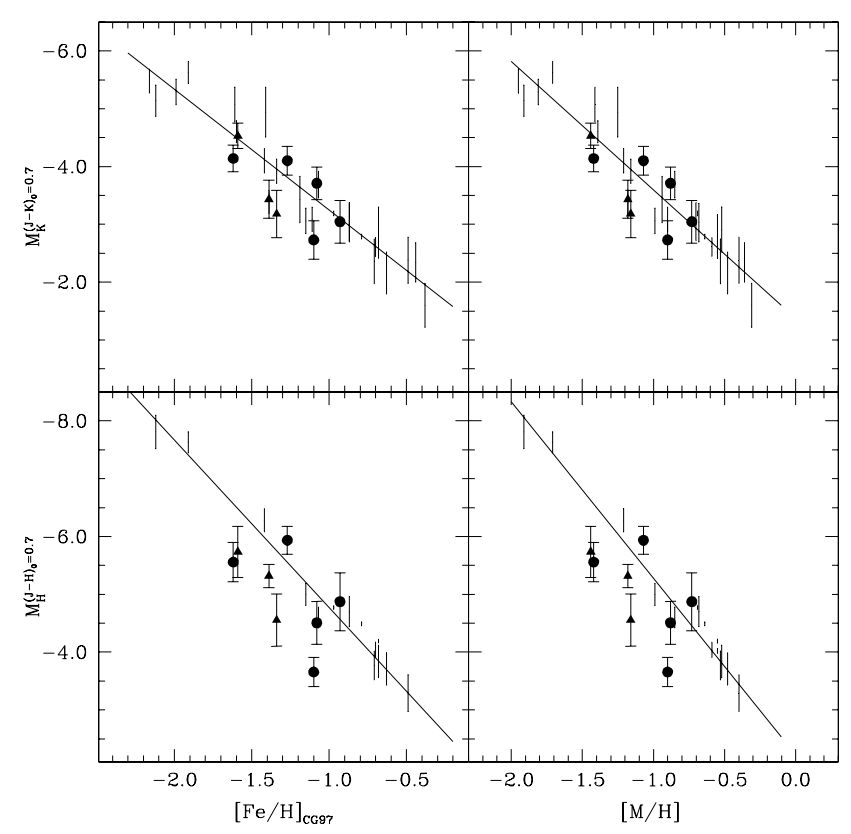

Fig. 7. RGB magnitude indices $M_{K}$ and $M_{H}$ at fixed color $(J-K)_{o}=$ $(J-H)_{o}=0.7$ as a function of the metallicity $[\mathrm{Fe} / \mathrm{H}]_{\mathrm{CG} 97}$ and the global metallicity $[\mathrm{M} / \mathrm{H}]$. Symbols are the same as Fig. 5.

decreasing cluster metallicity. We note here that the RGB slopes of NGC 6541 and NGC 6642 tend to be slightly flatter than the corresponding value in the previous calibration at the given metallicity, as shown in Fig. 8. Indeed, previous spectroscopic studies have pointed to those clusters as having low-tointermediate metallicities. Lee \& Carney (2002) obtained echelle spectra of two member stars in NGC 6541 and found the metallicity $[\mathrm{Fe} / \mathrm{H}]=-1.76 \pm 0.02$. Also, Rutledge et al. (1997) studied NGC 6541 using the Ca II triplet with low-resolution spectra and obtained $[\mathrm{Fe} / \mathrm{H}]=-1.79 \pm 0.02$. For NGC 6642, Minniti (1995) derived $[\mathrm{Fe} / \mathrm{H}]=-1.40 \pm 0.20$ from the spectroscopy of 13 giant stars selected from the near-infrared photometry. Therefore, we cautiously conclude that the near-infrared CMDs of NGC 6541 and NGC 6642 have somewhat flatter RGB slopes than those of the other clusters with similar metallicities.

\subsection{The luminosity function, the RGB bump and tip}

The LF of the RGB stars in a globular cluster provides the key observables of the CMD morphology, i.e., the RGB bump and the RGB tip. Each parameter has crucial astrophysical significance for the low-mass stellar evolution and the cosmic distance scale of the system.

The RGB bump is an important evolutionary feature that occurs during the post main-sequence evolution of low mass stars. Thomas (1967) and Iben (1968) pointed out the physical origin of the RGB bump. King et al. (1985) first identified the RGB bump from the observation of 47 Tuc. At the hydrogenburning shell stage after the first dredge-up in a star, when the convective envelope penetrates deeply enough into the narrow hydrogen burning shell, an evolutionary hesitation and a change in evolutionary rate is expected at some level along the RGB. This yields an RGB bump as a peak in the differential LF and a break in the slope of the cumulative LF of RGB stars in a cluster. The position in luminosity of the RGB bump in a globular cluster mainly depends on the metal abundance, helium abundance, cluster age, and other parameters that control the internal

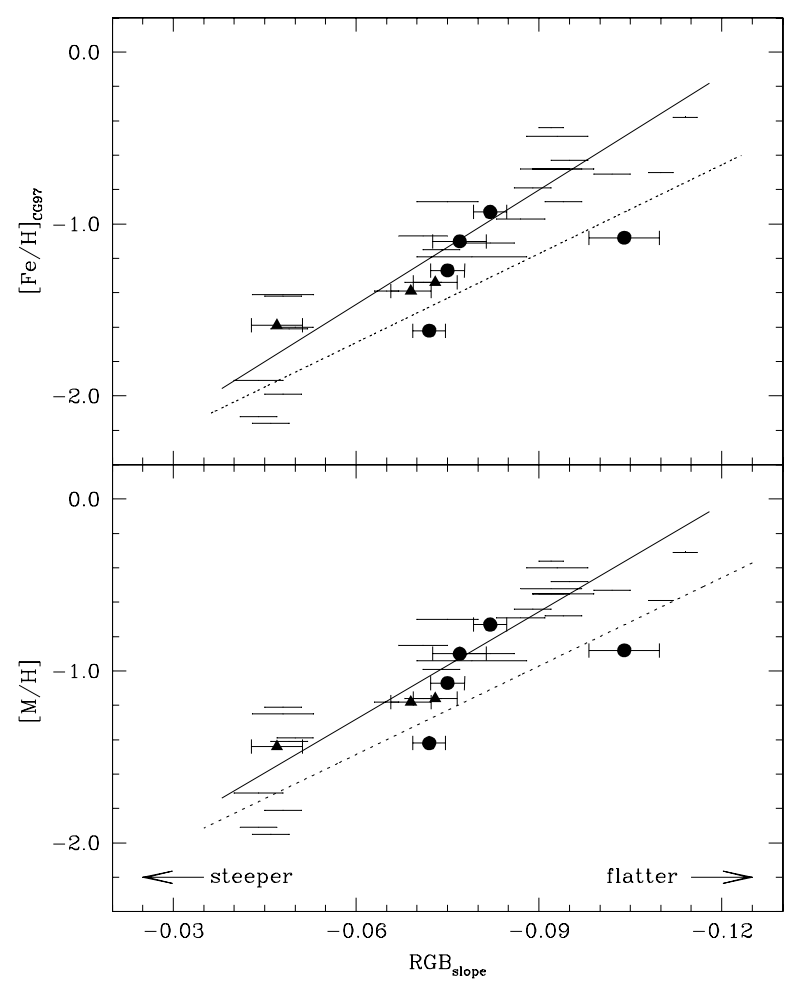

Fig. 8. The RGB slope as a function of metallicity. Symbols are the same as Fig. 5. The dotted lines are the relations found by Ivanov \& Borissova (2002).

convection of a star. The detection of the RGB bump from LFs has been the subject of many studies (Valenti et al. 2004b; Cho \& Lee 2002; Ferraro et al. 1999; Fusi Pecci et al. 1990; and references therein). Cho \& Lee (2002) measured the absolute $M_{K}$ magnitudes of the RGB bump for 16 globular clusters and suggested that the correlation between the $M_{K}$ of the RGB bump and the metallicity can be used to determine distance moduli of globular clusters with an uncertainty of $\Delta M_{K} \approx \pm 0.24$ mag at a given metallicity. Recently, Ferraro et al. (2000) and Valenti et al. (2004b) presented a new calibration of the relation between the cluster metallicity and the brightness of the RGB bump in the $K$ and bolometric magnitudes.

To construct the LFs of the RGB stars needed for an accurate measurement of the luminosity in $K$ of the RGB bump, we used the selected RGB stars to define the fiducial normal points of the $(J-K, K)$ CMDs for each cluster, as described in Sect. 3.3. To avoid contamination from the other populations, the selected RGB stars include only RGB samples within a $2 \sigma$ deviation of the mean color for a given magnitude bin. Figures 9 and 10 show the differential and cumulative LFs of RGB stars in three halo clusters and five metal-poor bulge clusters, respectively. The RGB bump is defined at a significant peak in the differential LF and a corresponding slope break in the cumulative LF for each cluster. Because the RGB sample size varied between clusters, we optimize the size of the magnitude bins for each cluster based on the number of samples. This enabled us to detect the RGB bump reliably regardless of sample size. Indeed, because the RGB bump luminosity increases with decreasing cluster metallicity and stars in metal-poor clusters have a high evolutionary rate at the end of the RGB, detecting the RGB bump is more difficult in metal-poor clusters than in metal-rich ones. The observed $K$, the absolute $M_{K}$ and the bolometric $M_{\text {bol }}$ magnitudes of the RGB bump for each cluster are listed in Table 4. 

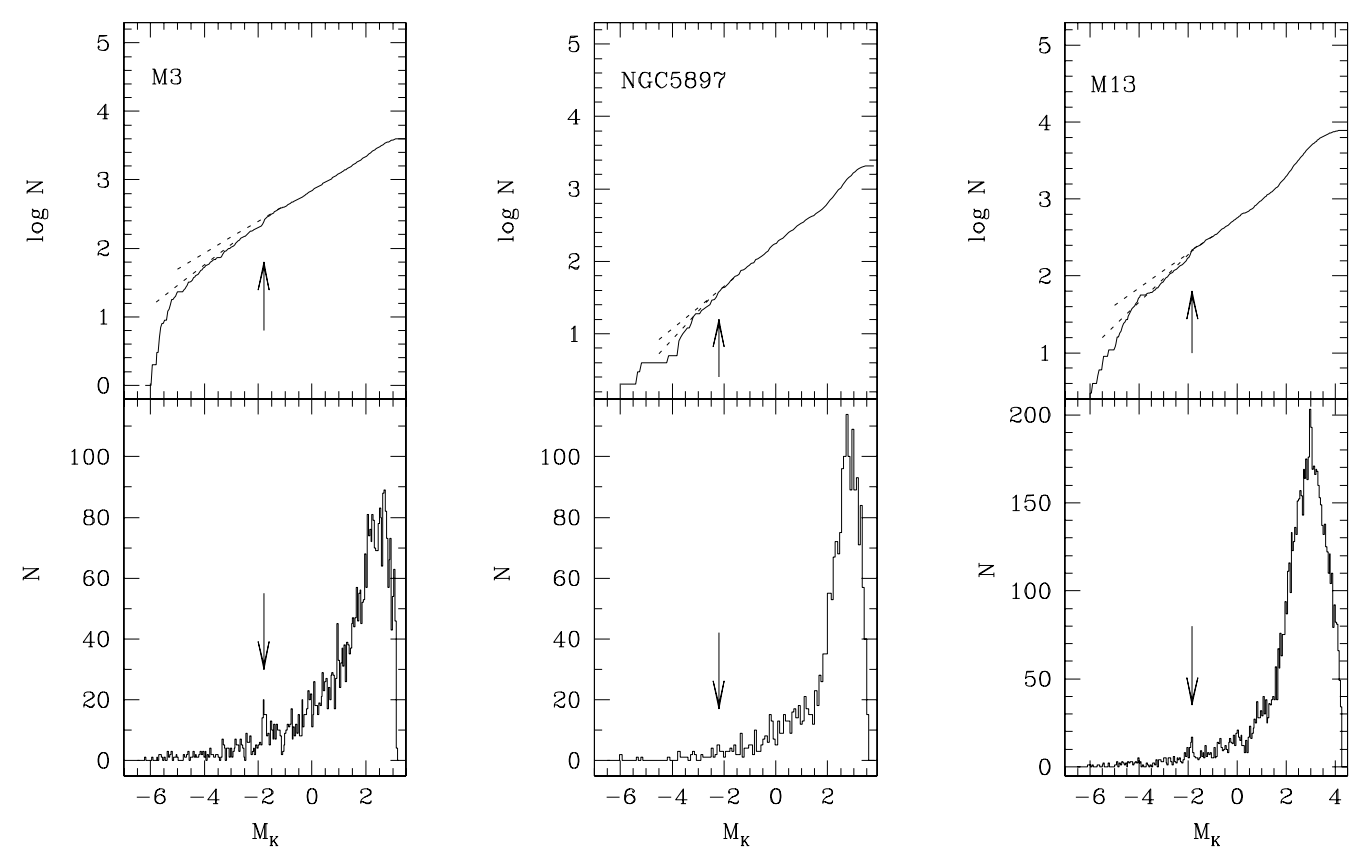

Fig. 9. The cumulative (upper) and differential (lower) LFs for RGB stars in the observed halo clusters. The arrows indicate the RGB bump position. The dashed lines in the cumulative LF are the linear fit to the regions above and below the RGB bump.
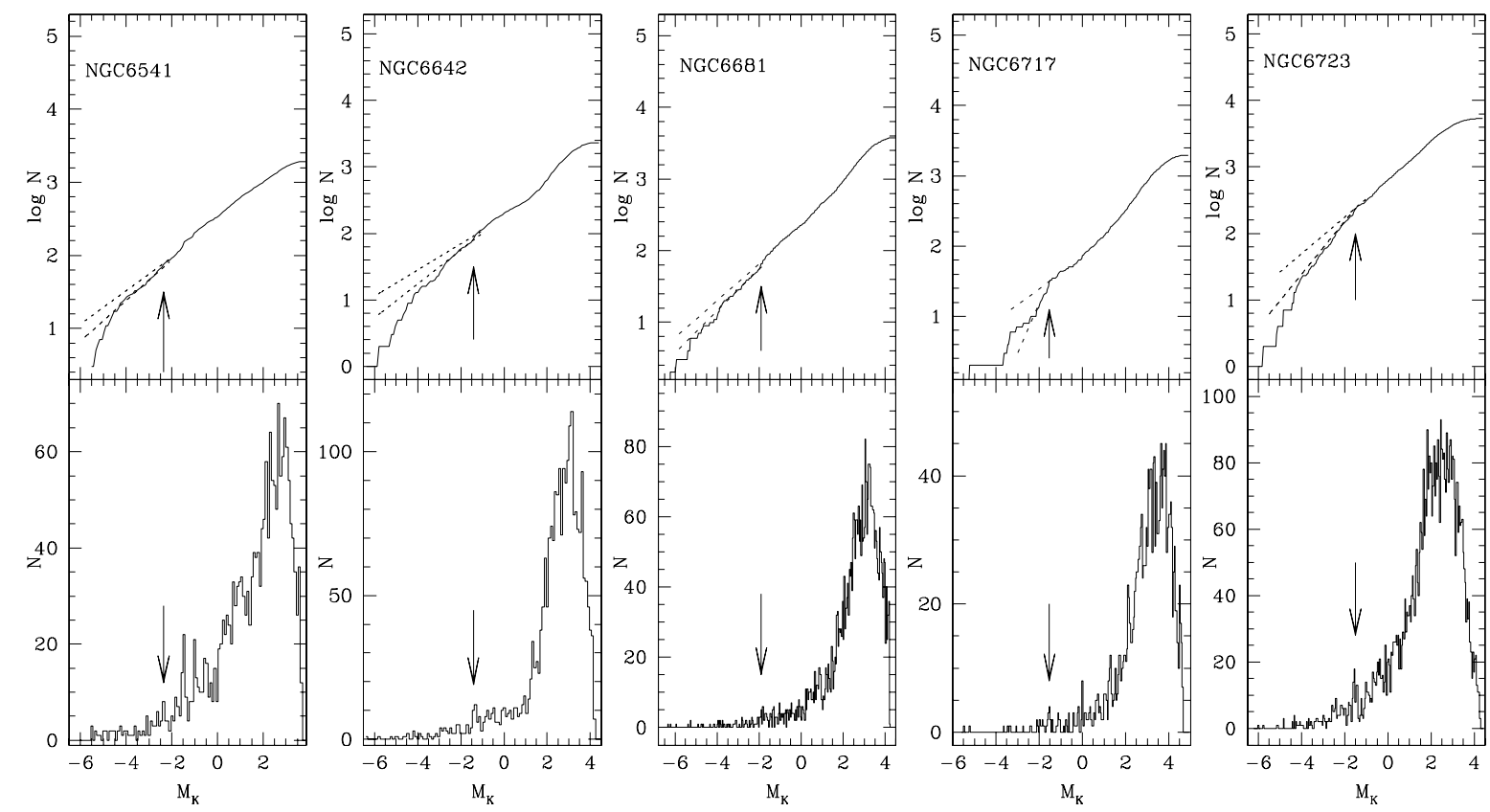

Fig. 10. As in Fig. 9 for the observed metal-poor bulge clusters.

Table 4. Photometric parameters of the RGB bump and tip for the observed clusters.

\begin{tabular}{ccccccccc}
\hline \hline Name & M3 & NGC 5897 & M 13 & NGC 6541 & NGC 6642 & NGC 6681 & NGC 6717 & NGC 6723 \\
\hline$K^{\text {Bump }}$ & $13.24 \pm 0.05$ & $13.45 \pm 0.08$ & $12.61 \pm 0.05$ & $11.74 \pm 0.09$ & $13.23 \pm 0.10$ & $13.13 \pm 0.04$ & $12.98 \pm 0.05$ & $13.19 \pm 0.05$ \\
$M_{K}^{\text {Bump }}$ & $-1.80 \pm 0.05$ & $-2.22 \pm 0.08$ & $-1.83 \pm 0.05$ & $-2.36 \pm 0.09$ & $-1.41 \pm 0.10$ & $-1.90 \pm 0.04$ & $-1.53 \pm 0.05$ & $-1.54 \pm 0.05$ \\
$M_{\text {Bol }}^{\text {Bump }}$ & $0.23 \pm 0.21$ & $-0.34 \pm 0.22$ & $0.15 \pm 0.21$ & $-0.48 \pm 0.22$ & $0.30 \pm 0.22$ & $-0.02 \pm 0.20$ & $0.57 \pm 0.21$ & $0.50 \pm 0.21$ \\
& & & & & & & & \\
$K^{\text {Tip }}$ & $8.83 \pm 0.05$ & $9.69 \pm 0.08$ & $8.36 \pm 0.05$ & $8.59 \pm 0.09$ & $8.29 \pm 0.10$ & $8.69 \pm 0.04$ & $9.02 \pm 0.05$ & $8.73 \pm 0.05$ \\
$M_{K}^{\text {Tip }}$ & $-6.21 \pm 0.05$ & $-5.98 \pm 0.08$ & $-6.08 \pm 0.05$ & $-5.67 \pm 0.09$ & $-6.35 \pm 0.10$ & $-6.34 \pm 0.04$ & $-5.49 \pm 0.05$ & $-6.00 \pm 0.05$ \\
$M_{\text {Bol }}^{\text {Tip }}$ & $-3.57 \pm 0.21$ & $-3.62 \pm 0.22$ & $-3.46 \pm 0.21$ & $-3.22 \pm 0.22$ & $-3.53 \pm 0.22$ & $-3.78 \pm 0.20$ & $-2.84 \pm 0.21$ & $-3.37 \pm 0.21$ \\
\hline
\end{tabular}




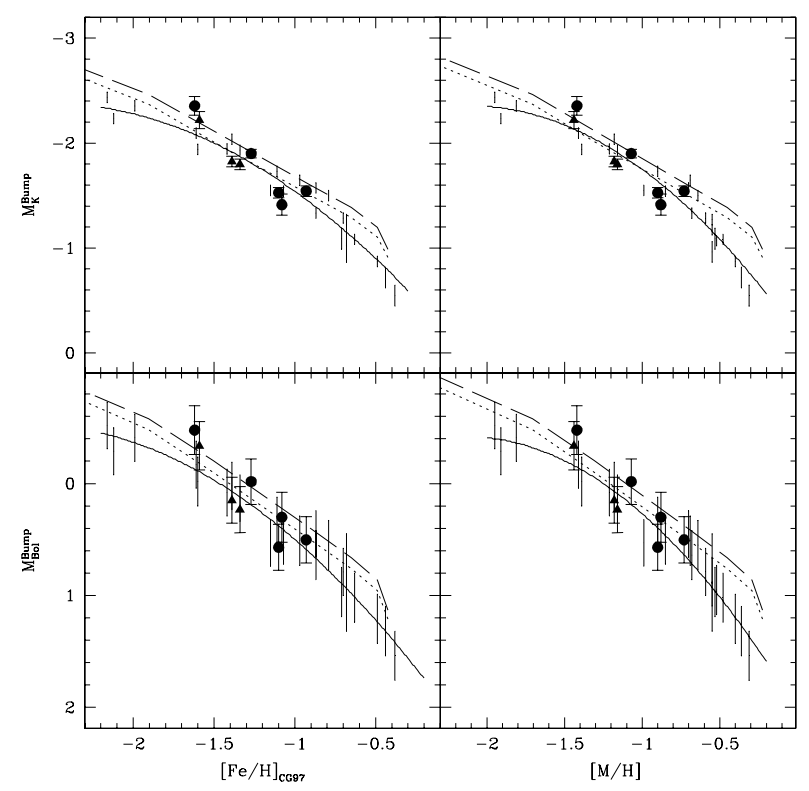

Fig. 11. The behavior of the $M_{K}$ and $M_{\mathrm{bol}}$ magnitudes of the RGB bumps for the observed clusters as a function of metallicity $[\mathrm{Fe} / \mathrm{H}]$ and global metallicity $[\mathrm{M} / \mathrm{H}]$. The dotted and dashed lines are the theoretical prediction of the Yonsei-Yale isochrone (Yi et al. 2003; Kim et al. 2002) at $t=12$ and $10 \mathrm{Gyr}$, respectively. The other symbols are the same as Fig. 5.

To convert the absolute magnitudes into bolometric magnitudes, we use the bolometric corrections for population II giants provided by Montegriffo et al. (1998). Figure 11 shows the behavior of the $M_{K}$ and $M_{\mathrm{bol}}$ magnitudes of the RGB bumps for each cluster as a function of metallicity $[\mathrm{Fe} / \mathrm{H}]_{\mathrm{CG} 97}$ and global metallicity $[\mathrm{M} / \mathrm{H}]$. The solid lines in Fig. 11 represent the calibration relation of Valenti et al. (2004b), which is consistent with the measured RGB bump magnitudes of the observed halo clusters and metal-poor bulge globular clusters. The dotted and dashed lines represent the theoretical prediction of the RGB bump magnitude as a function of metallicity from the Yonsei-Yale isochrone at $t=12$ and $10 \mathrm{Gyr}$ (Yi et al. 2003; Kim et al. 2002), indicating that the RGB bump moves to fainter locations with increasing cluster metallicity and age.

The luminosity of the RGB tip (TRGB) is a widely used standard candle to estimate the distance to galaxies of any morphological type (Walker 2003; Bellazzini et al. 2001; Ferrarese et al. 2000a,b; Madore \& Freedman 1995; Lee et al. 1993). In this paper, the magnitude of the TRGB was determined from brightness measurements of the brightest RGB stars, and the bright end of the observed LF of RGB stars (e.g., Sakai et al. 1996; Madore \& Freedman 1995). We note that the method is not statistically able to determine the TRGB because the brightest RGB is too poorly populated to define the TRGB in the limited area of the observed clusters. Here, we simply assume that the brightest RGB stars and the bright end of the LF of RGB stars represent the TRGB.

The measured $K, M_{K}$ and $M_{\mathrm{bol}}$ magnitudes of the TRGB for each cluster are listed in Table 4. Errors in $K$ and $M_{K}$ are measurement errors, and those in $M_{\mathrm{bol}}$ are a combination of measurement errors and the global uncertainty of the distance moduli, which is assumed to be $0.2 \mathrm{mag}$ (e.g., Cho \& Lee 2002). Frogel et al. $(1983,1981)$ found a well-defined relation between the bolometric luminosity of the brightest stars and the cluster metallicity. Recently, Ferraro et al. (2000) and Valenti et al. (2004b) presented a new calibration of the relation in the $K$

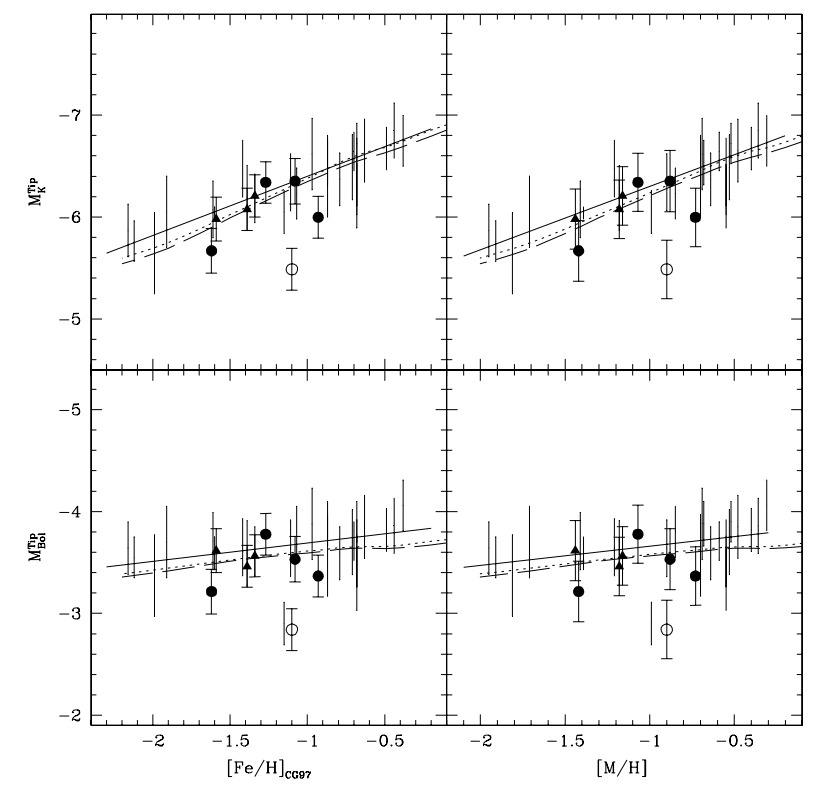

Fig. 12. As in Fig. 11 for the RGB tip. The open circles indicate the TRGB measurements of the post-core-collapse cluster NGC 6717.

and bolometric magnitudes. Figure 12 shows our measurements of $M_{K}$ and $M_{\mathrm{bol}}$ of the TRGB for the observed clusters in comparison with the other measurements and the calibration relation of Valenti et al. (2004b). It is also apparent in Fig. 12 that the theoretical prediction of the TRGB magnitude as a function of metallicity by the Yonsei-Yale isochrones (Yi et al. 2003; Kim et al. 2002) is in good agreement with the observations. We note that the open circles in Fig. 12 are the measurements of the TRGB magnitude of NGC 6717. NGC 6717 is a very concentrated post-core-collapse cluster (Trager et al. 1995), for which we detected a too small number of bright RGB stars to measure the TRGB on the observed CMD (cf. Piotto et al. 2002; Ortolani et al. 1999; Brocato et al. 1996).

\section{Summary}

We have used high quality near-infrared $J, H$, and $K^{\prime}$ images to investigate the CMD morphology of five metal-poor globular clusters in the Galactic bulge and three halo clusters. Photometric parameters to describe the RGB shape have been measured from the obtained near-infrared CMDs of the observed clusters. The results are summarized as follows:

1. The near-infrared CMDs obtained are moderately deep to detect the base of RGB at $\Delta K^{\prime} \sim \Delta H \approx 8$ mag fainter than the RGB tip, and $\sim 4$ mag below the HB. The SGB and the MSTO are also detectable in CMDs for most of the observed clusters.

2. The RGB colors at fixed magnitudes of $M_{K}=M_{H}=$ $(-5.5,-5,-4$ and -3$)$ and the RGB magnitudes at fixed colors of $(J-K)_{o}=(J-H)_{o}=0.70$ have been measured from the fiducial normal points of the CMDs. The indices of the observed metal-poor bulge clusters and halo clusters are in good agreement with the previous empirical calibrations of the RGB magnitudes and colors as a function of cluster metallicity for the halo clusters and metal-rich bulge globular clusters.

3. The RGB slope in $(J-K, K)$ plane has also been measured from the fiducial normal points at the magnitude range 
between 0 and $5 \mathrm{mag}$ fainter than the brightest point. The dependence of the RGB slopes on the metallicity for the observed halo and metal-poor bulge clusters is consistent with previous results, i.e., the RGB slope becomes progressively steeper with decreasing cluster metallicity.

4. The absolute magnitude of the RGB bump in $M_{K}$ is measured at a significant peak and a corresponding break of the differential and cumulative LFs for selected RGB stars of the observed clusters. The RGB tip magnitudes are also measured from the brightness measurements of the brightest RGB stars and LFs. The correlations between the cluster metallicity and the $M_{K}$ and $M_{\text {bol }}$ of the RGB bump and tip for the observed metal-poor bulge clusters and halo clusters are in good agreement with the previous empirical calibrations for the halo and metal-rich bulge globular clusters and the theoretical prediction of the Yonsei-Yale isochrone.

Acknowledgements. The authors thank the anonymous referee for the substantial comments that helped to improve the paper. This work was supported by grant No. R01-2006-000-10716-0 from the Basic Research Program of the Korea Science \& Engineering Foundation, for which we are grateful. Y. C. K. is supported by the Astrophysical Research Center for the Structure and Evolution of the Cosmos (ARCSEC) of Korea Science and Engineering Foundation (KOSEF) through the Science Research Center (SRC) program.

\section{References}

Alcaino, G., Liller, W., Alvarado, F., et al. 1997, AJ, 114, 2638 Alcaino, G., Liller, W., Alvarado, F., et al. 1999, A\&AS, 136, 461 Barbuy, B., Bica, E., Ortolani, S., \& Bonatto, C. 2006, A\&A, 449, 1019

Barbuy, B., Ortolani, S., \& Bica, E. 1999, A\&A, 348, 783

Bellazzini, M., Ferraro, F. R., \& Pancino, E. 2001, ApJ, 556, 635

Brocato, E., Buonanno, R., Malakhova, Y., \& Piersimoni, A. M. 1996, A\&A, 311,778

Carpenter, J. M. 2001, AJ, 121, 2851

Carreta, E., \& Gratton, R. G. 1997, A\&AS, 121, 95

Cho, D.-H., \& Lee, S.-G. 2002, AJ, 124, 977

Cohen, J. G., Frogel, J. A., \& Persson, S. E. 1978, ApJ, 222, 165

Côte, P. 1999, AJ, 118, 406

Da Costa, G. S., \& Armandroff, T. E. 1990, AJ, 100, 162

Davidge, T. J. 2000, AJ, 120, 1853

Davidge, T. J., \& Courteau, S. 1999, AJ, 117, 1297

Dinescu, D. I., Girard, T. M., van Altena, W. F., \& López, C. E. 2003, AJ, 125, 1373

Ferrarese, L., Ford, H. C., Huchra, J., et al. 2000a, ApJS, 128, 431

Ferrarese, L., Mould, J. R., Kennicutt, R. C., et al. 2000b, ApJ, 529, 745

Ferraro, F. R., Messineo, M., Fusi Pecci, F., et al. 1999, AJ, 118,1738

Ferraro, F. R., Montegriffo, P., Origlia, L., \& Fusi Pecci, F. 2000, AJ, 119, 1282

Frogel, J. A., Persson, S. E., \& Cohen, J. G. 1981, ApJ, 246, 842
Frogel, J. A., Cohen, J. G., \& Persson, S. E. 1983, ApJ, 275, 773

Fusi Pecci, F., Ferraro, F. R., Crocker, D. A., Rood, R. T., \& Buonanno, R. 1990, A\&A, 238. 95

Harris, W. E. 1996, AJ, 112, 1487

Iben, I. Jr. 1968, Nature, 220, 143

Ivanov, V. D., \& Borissova, J. 2002, A\&A, 390, 937

Kang, A., Sohn, Y. -J., Rhee, J., et al. 2005, A\&A, 437, 61

Kim, Y.-C., Demarque, P., Yi, S. K., \& Alexander, D. R. 2002, ApJS, 143, 499

King, C. R., Da Costa, G. S., \& Demarque, P. 1985, ApJ, 299, 674

Kuchinski, L. E., \& Frogel, J. A. 1995, AJ, 110, 2844

Kuchinski, L. E., Frogel, J. A., Terndrup, D. M., \& Persson, S. E. 1995, AJ, 109, 1131

Lee, J.-W., \& Carney, B. W. 2002, AJ, 124, 1511

Lee, M. G., Freedman, W. L., \& Madore, B. F. 1993, ApJ, 417, 553

Lee, Y.-W., Demarque, P., \& Zinn, R. 1994, ApJ, 423, 248

Madore, B. F., \& Freedman, W. L. 1995, AJ, 109, 1645

McWilliam, A., \& Rich, R. M. 1994, ApJS, 91, 749

Minniti, D. 1995, A\&A, 468, 474

Minniti, D. 1996, ApJ, 459, 175

Montegriffo, P., Ferraro, F. R. Origlia, L., \& Fusi Pecci, F. 1998, MNRAS, 297, 872

Ortolani, S. 1999, Ap\&SS, 265, 355

Ortolani, S., Barbuy, B., \& Bica, E. 1991, A\&A, 249, 31

Ortolani, S., Barbuy, B., \& Bica, E. 1999, A\&AS, 136, 237

Ortolani, S., Momany, Y., Bica, E., \& Barbuy, B. 2000, A\&A, 357, 495

Ortolani, S., Bica, E., \& Barbuy, B. 2003, A\&A, 402, 565

Piotto, G., King, I. R., Djorgovski, S. G., et al. 2002, A\&A, 391, 945

Rieke, G. H., \& Lebofsky, M. J. 1985, ApJ, 288, 618

Rutledge, G. A., Hesser, J. A., \& Stetson, P. B. 1997, PASP, 109, 907

Sakai, S., Madore, B. F., \& Freedman, W. L. 1996, ApJ, 461, 713

Salaris, M., Chieffi, A., \& Straniero, O. 1993, ApJ, 414, 580

Schlegel, D. J., Finkbeiner, D. P., \& Davis, M. 1998, ApJ, 500, 525

Simons, D. A., \& Tokunaga, A. 2002, PASP, 114, 169

Sohn, Y.-J., Kang, A., Rhee, J., et al. 2006, A\&A, 445, 69

Sollima, A., Ferraro, F. R., Origlia, L., Pancino, E., \& Bellazzini, M. 2004, A\&A, 420, 173

Stetson, P. B. 1987, PASP, 99, 191

Stetson, P. B., \& Harris, W. E. 1988, AJ, 96, 909

Straniero, O., Chieffi, A., \& Limongi, M. 1997, ApJ, 490, 425

Thomas, H.-C. 1967, Z. Ap., 67, 420

Tokunaga, A. T., Simons, D. A., \& Vacca, W. D. 2002, PASP, 114, 180

Trager, S. C., King, I. R., \& Djorgovski, S. 1995, AJ, 109, 218

Valenti, E., Ferraro, F. R., \& Origlia, L. 2004a, MNRAS, 351, 1204

Valenti, E., Ferraro, F. R., \& Origlia, L. 2004b, MNRAS, 354, 815

Valenti, E., Ferraro, F. R., Perina, S., \& Origlia, L. 2004c, A\&A, 419, 139

Valenti, E., Origlia, L. \& Ferraro, F. R. 2005, MNRAS, 361, 272

Wainscoat, R., \& Cowie, L. 1992, AJ, 103, 332

Walker, A. R. 2003, in Stellar candles for the extragalactic distance scale, ed. D. Alloin, \& W. Gieren (Springer), Lect. Notes Phys., 635, 265

Yi, S., Demarque, P., \& Kim, Y.-C. 1997, ApJ, 482, 677

Yi, S. K., Kim, Y.-C., \& Demarque, P. 2003, ApJS, 144, 259

Zinn, R. J. 1985, ApJ, 293, 424

Zoccali, M., Renzini, A., Ortolani, S., et al. 2003, A\&A, 399, 931 